\title{
First observations with SuperCam and future plans
}

\author{
Jenna Kloosterman ${ }^{a}$, Tiara $\operatorname{Cottam}^{a}$, Brandon Swift $^{a}$, David Lesser ${ }^{a}$, Paul Schickling ${ }^{a}$, \\ Christopher Groppi ${ }^{b}$, Michael Borden ${ }^{a}$, Allison Towner ${ }^{a}$, Per Schmidt ${ }^{a}$, Craig Kulesa ${ }^{a}$, \\ Christian d'Aubigny ${ }^{a}$, Christopher Walker $^{a}$, Dathon Golish ${ }^{a}$, Sander Weinreb ${ }^{c}$, Glenn Jones ${ }^{c}$, \\ Hamdi Mani ${ }^{b}$, Jacob $\mathrm{Kooi}^{c}$, Art Lichtenberger ${ }^{d}$, Patrick Puetz ${ }^{e}$, Gopal Narayanan ${ }^{f}$ \\ ${ }^{a}$ Steward Observatory, University of Arizona, Tucson, AZ 85721, USA; \\ ${ }^{b}$ School of Earth and Space Exploration, Arizona State University, Tempe, AZ 85287, USA; \\ ${ }^{c}$ Department of Electrical Engineering, California Institute of Technology, Pasadena, CA \\ 91125, USA; \\ ${ }^{d}$ Department of Electrical Engineering, University of Virginia, Charlottesville, VA 22908, USA; \\ ${ }^{e}$ KOSMA, University of Cologne, Cologne, Germany; \\ fDepartment of Astronomy, University of Massachusetts, Amherst, MA 01003;
}

\begin{abstract}
Supercam is a $345 \mathrm{GHz}$, 64-pixel heterodyne imaging array for the Heinrich Hertz Submillimeter Telescope (HHSMT). By integrating SIS mixer devices with Low Noise Amplifiers (LNAs) in $8-1 \mathrm{x} 8$ pixel modules, the size needed for the cryostat and the complexity of internal wiring is significantly reduced. All subsystems including the optics, cryostat, bias system, IF boxes, and spectrometer have been integrated for all 64 pixels. In the spring of 2012, SuperCam was installed on the HHSMT for an engineering run where it underwent system level tests and performed first light observations. In the fall of 2012 SuperCam will begin a 500 square degree survey of the Galactic Plane in ${ }^{12} \mathrm{CO} \mathrm{J}=3-2$. This large-scale survey will help answer fundamental questions about the formation, physical conditions, and energetics of molecular clouds within the Milky Way. The data set will be available via the web to all interested researchers.
\end{abstract}

Keywords: submillimeter, heterodyne, array receiver

\section{INTRODUCTION}

SuperCam is a 64 pixel, Superheterodyne Camera designed to operate in the $345 \mathrm{GHz}$ atmospheric window. Its development was motivated by the need to perform large-scale, high spectral resolution submillimeter spectroscopy of the interstellar medium (ISM).

SuperCam's 1x8 integrated mixer modules were fabricated out of 145 copper alloy at the University of Arizona using a Kern MMP micromilling machine. SuperCam integrates multiple Superconductor-InsulatorSuperconductor (SIS) mixing devices and Microwave Monolithic Integrated Circuit (MMIC) Low Noise Amplifiers (LNAs) into the same waveguide mixer block. Tests of the initial prototype mixer block showed that there is no penalty in performance as a result of this integration. The LNAs are stable and do not overheat the SIS devices. ${ }^{1}$ The SIS devices have been optimized to work between 320-380 GHz. An array of diagonal feedhorns couples the emergent beams to the arrays optical system. ${ }^{2}$

Previous work on SuperCam included noise temperature measurements of 29 pixels. $^{2,3}$ All 64 pixels were installed in the cryostat in 2011 and a test tone with 8 pixels was recorded. ${ }^{4}$ Since the last update, the cryostat, bias system, IF down-converters and spectrometer have been integrated and tested in our laboratory at the University of Arizona. SuperCam was taken to the Heinrich Hertz Submillimeter Telescope (HHSMT) for an engineering run and saw first light on May 24, 2012. For this run the instrument was configured with 32 operational pixels. Here we summarize the science $(\S 1.1)$ and instrument design (§1.2). The results of the laboratory tests are in $\S 2$ and the results of the engineering run are in $\S 3$.

Millimeter, Submillimeter, and Far-Infrared Detectors and Instrumentation for Astronomy VI, edited by Wayne S. Holland, Jonas Zmuidzinas, Proc. of SPIE Vol. 8452,

845204 @ 2012 SPIE $\cdot$ CCC code: 0277-786X/12/\$18 · doi: 10.1117/12.925088

Proc. of SPIE Vol. $8452845204-1$ 


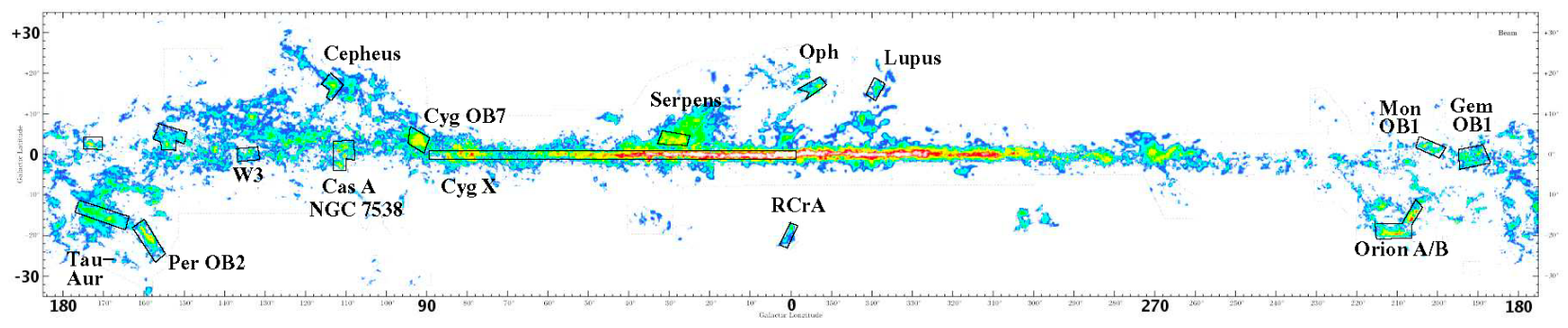

Figure 1. The 500 square degree SuperCam Galactic Plane Survey from the HHSMT has been outlined in black. The survey will Nyquist sample the galactic plane from $0^{\circ}<l<90^{\circ},-1^{\circ}<b<+1^{\circ}$ and all major star forming regions visible from the HHSMT.

\subsection{Science}

SuperCam will map 500 square degrees in the Galactic Plane between $0^{\circ}<l<90^{\circ}$ and $-1<b<+1$ and all major star-forming regions visible from the HHSMT with 23 " spatial resolution and $0.3 \mathrm{~km} / \mathrm{s}$ velocity resolution. This survey has been outlined in black in Figure 1. The SuperCam survey will improve upon the spatial resolution of existing Galactic Plane surveys by a factor $>10$. The depth of the survey is sufficient to detect CO to a level consistent with $\mathrm{A}_{\nu} \sim 1$, detecting all $\mathrm{CO}$ that has formed in-situ. ${ }^{3}$ By combining the results of the SuperCam survey in ${ }^{12} \mathrm{CO} \mathrm{J}=3-2$ and ${ }^{13} \mathrm{CO} \mathrm{J}=3-2$ with existing $\mathrm{CO} \mathrm{J}=1-0$ surveys, an excitation temperature map of the survey region can be constructed. The SuperCam survey will provide valuable insights into the dynamics and energetics of the interstellar medium. ${ }^{2-4}$

\subsection{Instrument Description}

SuperCam is composed of several subsystems: optics, cryogenics, and electronics. A block diagram of the instrument is provided in Figure 2. A recent photograph of SuperCam's subsystems fully cabled in the lab is shown in Figure 3. The instrument consists of 1x8 arrays that are stacked 8 wide for a total of 64 pixels (see Figure 4). Inside each pixel, waveguide directs the incoming sky signal and Local Oscillator (LO) around a $90^{\circ}$ curve to the SIS junction. The LO consists of a single Virginia Diodes, Inc. (VDI) solid-state source. A corporate waveguide power divider is used to split the output from the source into 64 independent beams with enough LO power to pump each pixel. A simple Mylar beam splitter is used to combine signal and LO beams.

The IF output signal of each SIS device is directly wire bonded onto a MMIC LNA. With only $8 \mathrm{~mW}$ of power, each MMIC LNA achieves a $\sim 5 \mathrm{~K}$ noise temperature and provides $32 \mathrm{~dB}$ of gain. ${ }^{2,3} \mathrm{The} 5 \mathrm{GHz} \mathrm{IF}$ output signal is then amplified further and downconverted to baseband in an IF Processor Box. There is an individual IF Processor Box for each 1x8 mixer array. Each of SuperCams subsystems has been documented in previous papers. ${ }^{2-4}$ End-to-end tests of these systems were performed in the lab before installation on the HHSMT.

\section{LABORATORY RESULTS}

We have completed the fabrication and assembly of all 64 pixels. The bias and pre-amp boxes have been fully tested and are working as expected. The optical relay system uses 7 direct machined aluminum mirrors. The mirrors have been polished to within a few microns rms and nickel-plated to allow laser alignment. This system is used to convey and transform the $\mathrm{f} \# 13.8$ beam from the HHSMT secondary to the $\sim \mathrm{f} \# 6.5$ beams of the mixer arrays. These beam sizes have been verified to first order with beam pattern measurements.

There are currently 46 live devices, 61 working LNAs, and 61 working magnets, for a total of 40 fully operational pixels. Figure 5 shows a histogram of noise temperatures for all working pixels. The devices were selected at random from a UVa wafer. There are 32 pixels with noise temperatures better than 150 K. These 32 pixels were used on the recent SuperCam test run. The yield of high sensitivity devices will be increased via a device characterization and replacement campaign in summer 2012.

Further author information: (Send correspondence to J.L.K)

J.L.K.: E-mail: jlkloost@email.arizona.edu, Telephone: 15206212026 


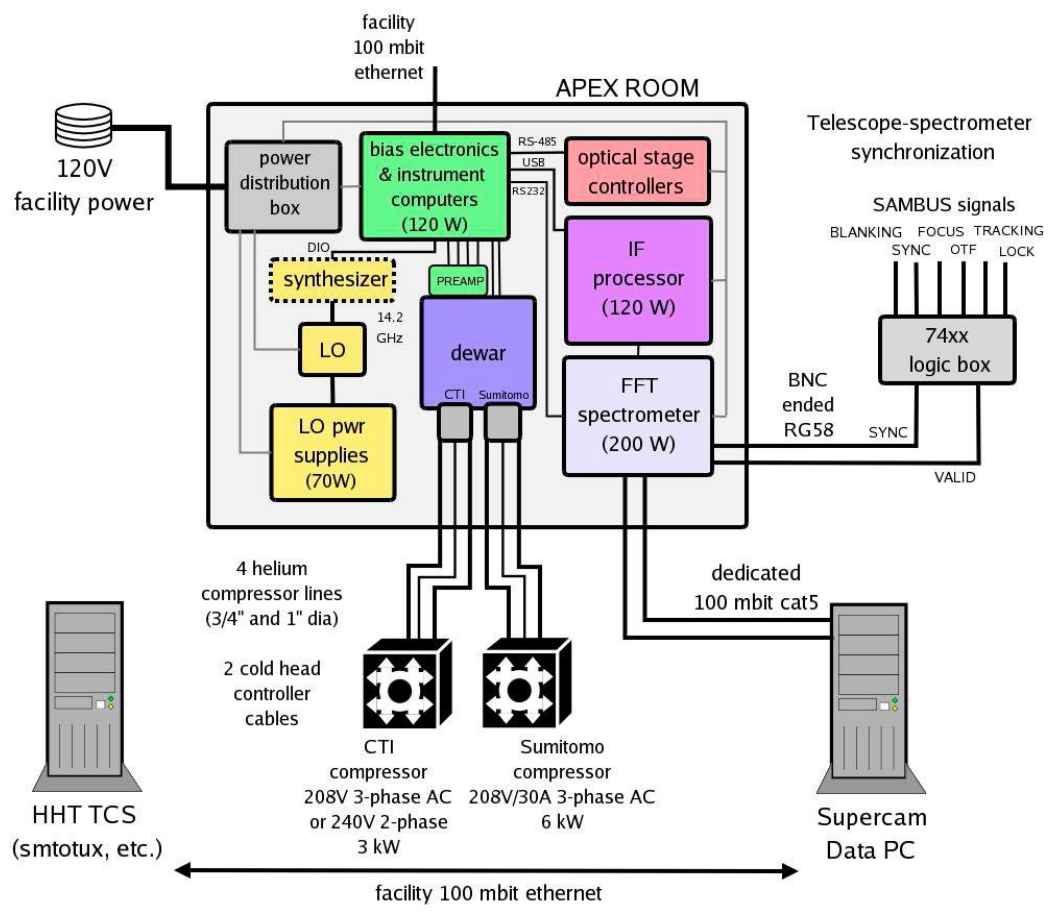

Figure 2. A block diagram of SuperCam. The cryostat, pre-amplifier electronics, bias electronics, LO, IF downconverters, spectrometer, power supplies, control computers reside in the HHSMT's Apex room, located just behind the $10 \mathrm{~m}$ dish.

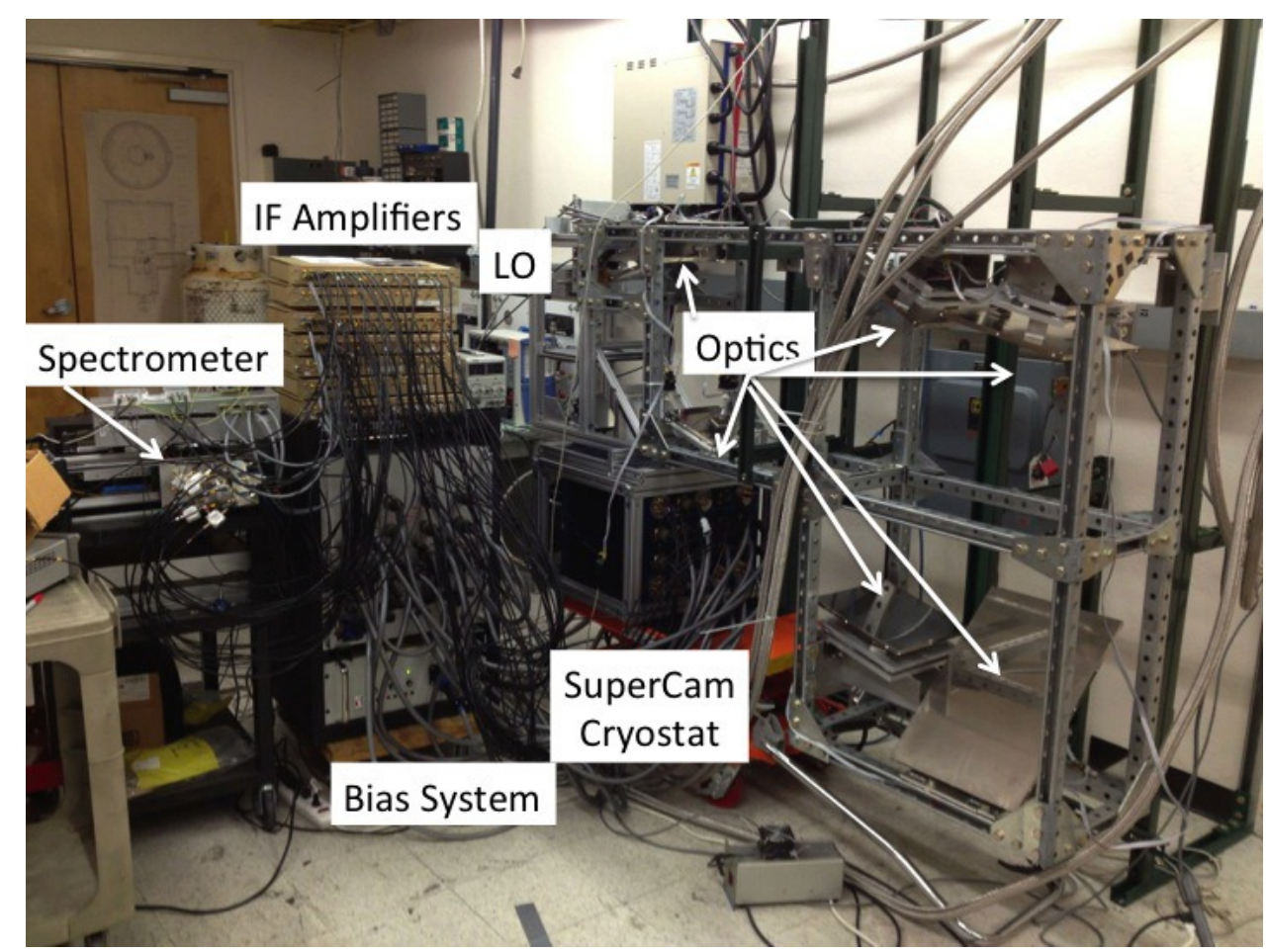

Figure 3. Photograph of the SuperCam cryostat, LO, and electronics integrated at the University of Arizona, AZ. 


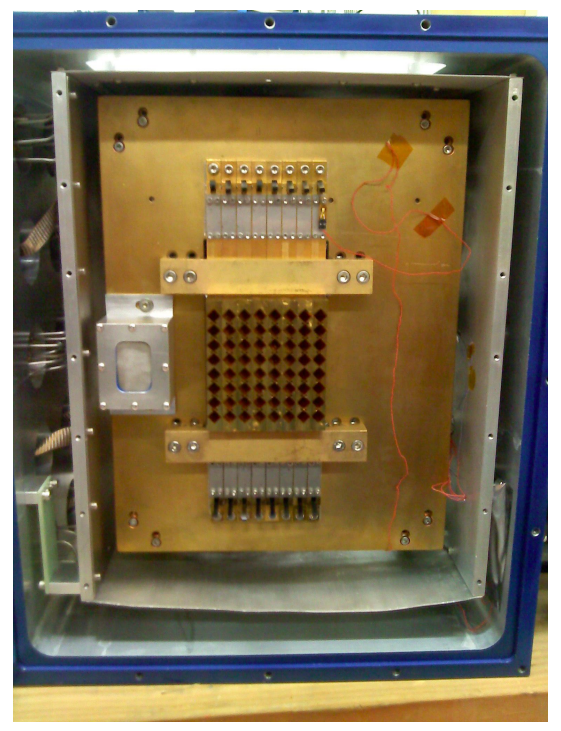

Figure 4. An array of 64 feedhorns directs incoming signal to 64 waveguides machined in 8 - $1 x 8$ blocks. All 64 pixels were installed in the SuperCam cryostat in April 2011.

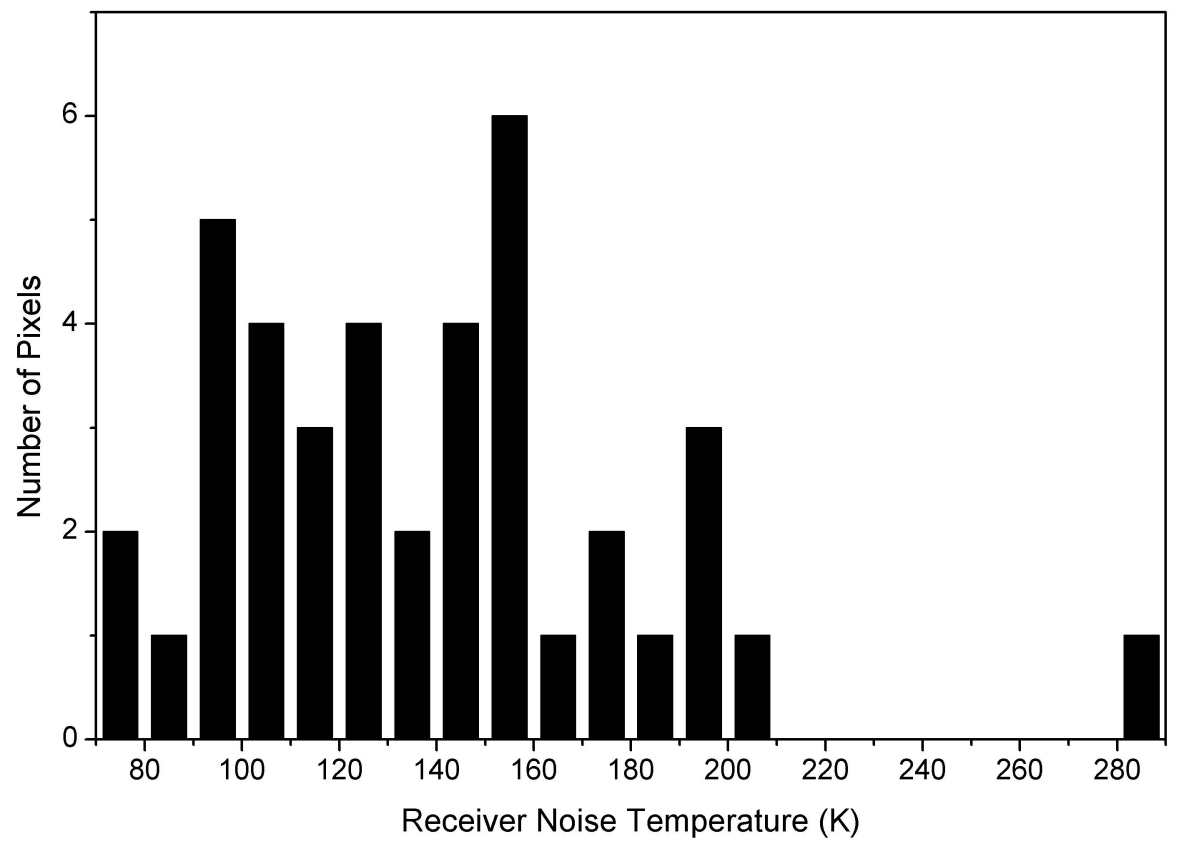

Figure 5. Histogram of noise temperatures for the 40 working pixels installed in SuperCam. 


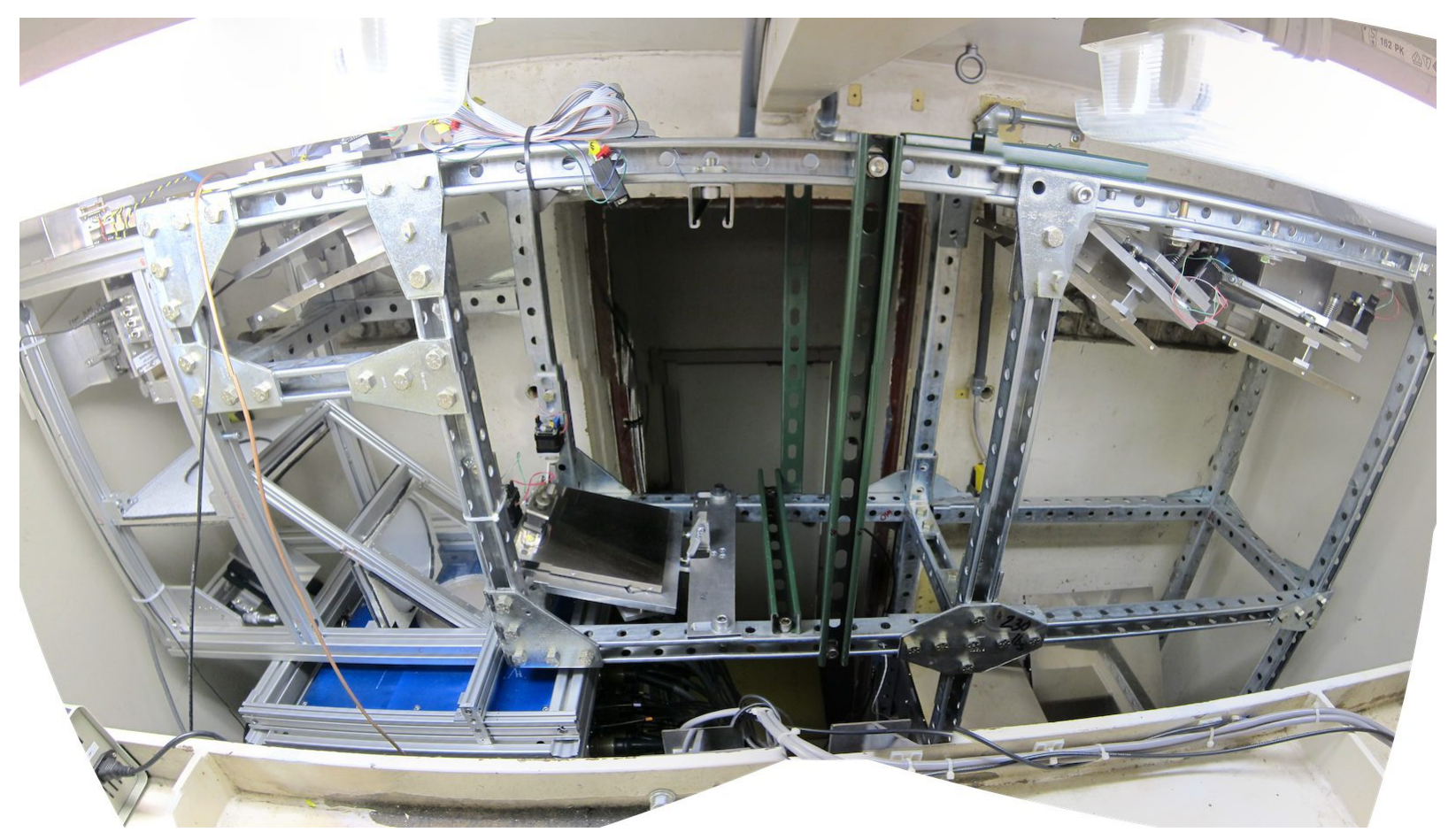

Figure 6. This photograph shows a fisheye panoramic view of SuperCam mounted in the Apex Room of the HHSMT.

\section{ENGINEERING RUN}

\subsection{Installation}

Installation of SuperCam on the HHSMT began at the beginning of May 2012. A fisheye panoramic photograph of the SuperCam cryostat, local oscillator and optical relay system mounted in the Apex Room is shown in Figure 6 . The LO can be seen in the top left-hand corner with its corresponding optics down the left-hand side. The SuperCam cryostat is aligned to the incoming light from the telescope via the optical relay system. These mirrors are mounted on an optical support structure that is bolted to the back wall. When SuperCam is in operation a pick-off mirror captures the telescope beam from the tertiary and directs it to the instrument's relay optics.

\subsection{First Light}

After $\sim 2$ weeks of testing and integration, SuperCam saw first light with 32 pixels on May 24, 2012. From measurements on Mars and Saturn, the main beam efficiency of the array pixels was found to be $\sim 60 \%$, with a $3 \mathrm{~dB}$ beam size of $\sim 23^{\prime \prime}$. Figure 7 shows a $10^{\prime} \times 10^{\prime}{ }^{12} \mathrm{CO}$ map of DR21 taking during the first test run. The DR21 HII region is part of the Cygnus X complex of molecular clouds, which hosts a large number of massive star forming cores. DR21 in particular is a portion of a north-south oriented chain of massive young stellar objects (YSOs) seen in the mm wave dust continuum, water masers, and high-J CO emission. In this map, the elongated east-west massive outflow of DR21 is seen prominently in ${ }^{12} \mathrm{CO} \mathrm{J}=3-2$ emission; the western side shows a highly collimated flow, whereas the eastern side is blistered and difficult to distinguish from the ambient cloud. The structure of the $\mathrm{J}=3-2$ emission in the DR21 outflow follows the highly excited $\mathrm{H}_{2}$ emission seen in the near-infrared. After a break in the ridge just to the north of DR21, the warm dense gas peaks again at $3^{\prime}$ north, where it is thought that the clouds harboring DR21 and W75 to the north are interacting.

\section{FUTURE WORK}

After its engineering run, SuperCam was removed from the Apex room of the HHSMT. Over the summer, the cryostats refrigerators will be refurbished and the remaining pixels made fully operational. The 500 square degree 


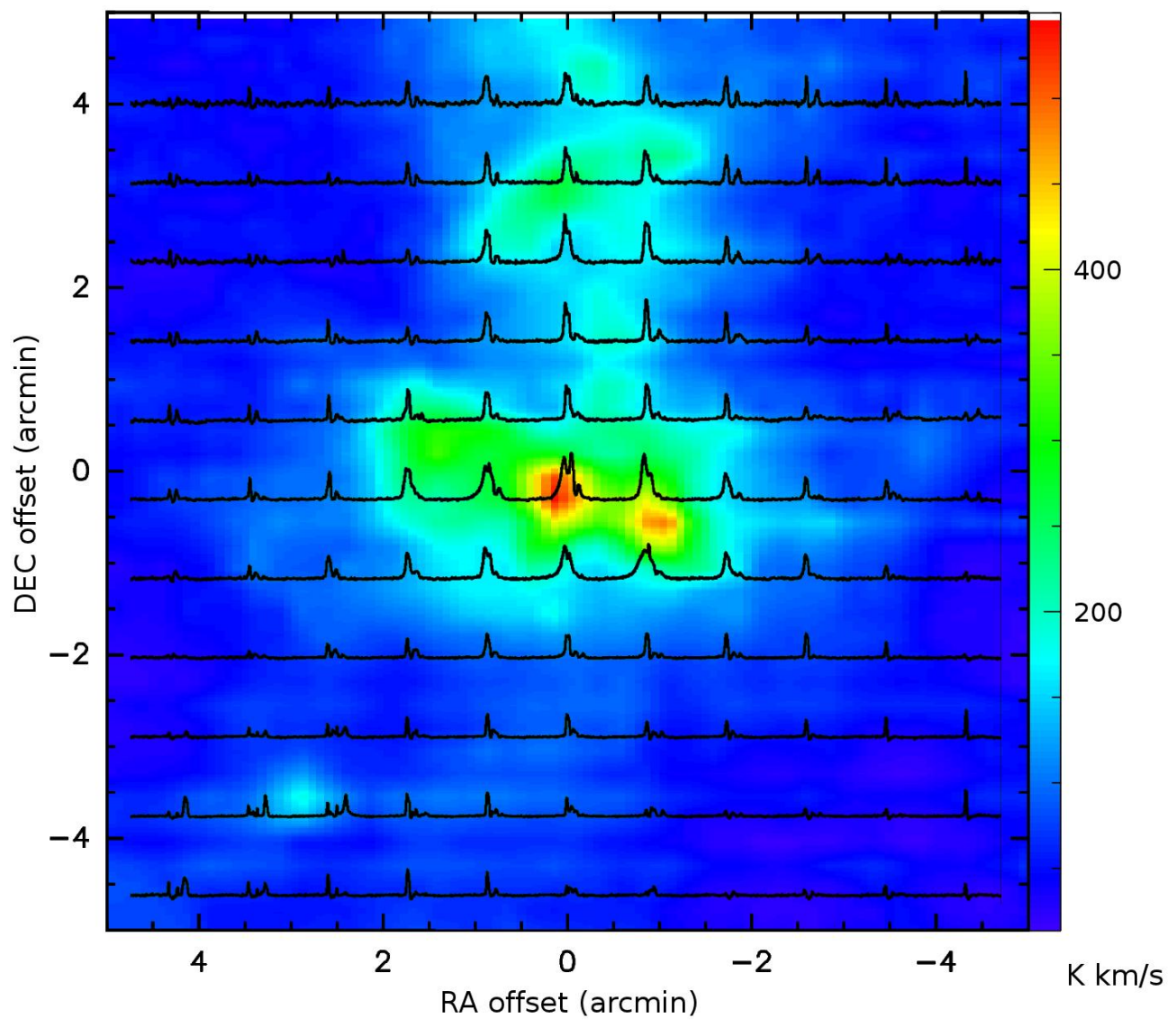

Figure 7. This map of the massive star forming region DR21 depicts the integrated intensity (in K $\mathrm{km} / \mathrm{s}$ units) of ${ }^{12} \mathrm{CO}$ $\mathrm{J}=3-2$ at $345.795 \mathrm{GHz}$. Binned spectra are overlaid atop the color map, each spanning the velocity range of -60 to 40 $\mathrm{km} / \mathrm{s}$, with a main beam brightness scale of 0 to $50 \mathrm{~K}$.

northern ${ }^{12} \mathrm{CO} \mathrm{J}=3-2$ Galactic Plane survey will begin this fall. Plans are being made to conduct a southern Galactic Plane survey with SuperCam beginning in 2014.

\section{CONCLUSIONS}

SuperCam is a leap forward in the realization of integrated heterodyne arrays for THz astronomy. SuperCam was installed on the HHSMT in May 2012, with first light observations commencing on May 24, 2012. A complete northern sky survey of the Milky Way in the ${ }^{12} \mathrm{CO} \mathrm{J}=3 \rightarrow 2$ line will begin in the fall. These maps will provide new, valuable insights into the life cycle of the ISM and the inner working of our Galaxy.

\section{ACKNOWLEDGMENTS}

We thank our project manager Brian Duffy and the Arizona Radio Observatory (ARO) staff for making this test run a success. The construction of SuperCam was supported by the NSF Major Research Instrumentation Program, Award AST- 0421499.

\section{REFERENCES}

[1] Puetz, P., Hedden, A., Gensheimer, P., Golish, D., Groppi, C. E., Kulesa, C., Narayanan, G., Lichtenberger, A., Kooi, J. W., Wadefalk, N., Weinreb, S., and Walker, C. K., "345 GHZ Prototype SIS Mixer with Integrated Mmic Lna," International Journal of Infrared and Millimeter Waves 27, 1365-1379 (Oct. 2006). 
[2] Groppi, C., Walker, C., Kulesa, C., Golish, D., Kloosterman, J., Weinreb, S., Jones, G., Barden, J., Mani, H., Kuiper, T., Kooi, J., and Lichtenberger, A., "Testing and Integration of Supercam, a 64- Pixel Array Receiver for the $350 \mathrm{GHz}$ Atmospheric Window," Twenty-First International Symposium on Space Terahertz Technology , 368-373 (Mar. 2010).

[3] Groppi, C., Walker, C., Kulesa, C., Golish, D., Kloosterman, J., Weinreb, S., Jones, G., Bardin, J., Mani, H., Kuiper, T., Kooi, J., Lichtenberger, A., Cecil, T., Puetz, P., Narayanan, G., and Hedden, A., "Test and integration results from SuperCam: a 64-pixel array receiver for the $350 \mathrm{GHz}$ atmospheric window," Proc. SPIE 7741, 77410X-77410X-12 (July 2010).

[4] Kloosterman, J., Groppi, C., Kulesa, C., Walker, C., Cottam, T., Ligget, E., Lesser, D., Borden, M., Schickling, P., Golish, D., d’Aubigny, C., Weinreb, S., Jones, G., Barden, J., Mani, H., Kooi, J., Lichtenberger, A., Cecil, P., Puetz, P., Narayanan, G., Hedden, A., and Xu, X., "A Progress Update on Supercam: A 345 GHz, 64-pixel Heterodyne Imaging Spectrometer," Twenty-Second International Symposium on Space Terahertz Technology , 174-178 (Apr. 2011). 